\title{
Reactivity and Molecular Recognition - Amine Methylation by an Introverted Ester
}

\author{
Byron W. Purse, Pablo Ballester, and Julius Rebek, Jr.* \\ The Skaggs Institute for Chemical Biology and Department of Chemistry, The Scripps Research \\ Institute, 10550 North Torrey Pines Rd, La Jolla, California 92037
}

\section{Supporting Information}

\section{Synthesis of new molecules.}

\section{General Experimental}

All reagents were obtained from commercial suppliers and used without further purification unless otherwise noted. Triethylamine was redistilled from $\mathrm{CaH}_{2}$ prior to use. Dichloromethane and THF were dried using columns of activated alumina according to the method of Grubbs et al. ${ }^{i}$ Other dry solvents were purchased as such and used directly. Reaction progress was monitored with thin layer chromatography on Merck $60 \mathrm{~F}_{254} 0.25 \mu \mathrm{m}$ silica plates. Flash chromatography was carried out using Silicycle R10030B $60 \AA$ $230-400$ mesh silica gel. ${ }^{1} \mathrm{H}$ and ${ }^{13} \mathrm{C}$ NMR spectra were obtained using a Bruker DRX-600 spectrometer at $300 \mathrm{~K}$ unless otherwise stated. Infrared absorption spectra were obtained using a Perkin-Elmer Paragon FT-IR spectrometer. MALDI-FTMS experiments were performed on an IonSpec FTMS mass spectrometer. ESI-MS experiments were performed on a single-quadrapole Perkin-Elmer API100 Sciex mass spectrometer or a Hewlett-Packard Series 1100 LC-MS.

\section{Synthetic Procedures}

Hexanitro cavitand 4. Ethyl-footed resorcin[4] $\operatorname{arene}^{\text {ii }}(2.4 \mathrm{~g}, 4.0 \mathrm{mmol})$ along with 1,2-difluoro4,5-dinitrobenzene $(2.45 \mathrm{~g}, 12.0 \mathrm{mmol})$ were placed in a $250 \mathrm{~mL} \mathrm{3-necked} \mathrm{flask} \mathrm{fitted} \mathrm{with} \mathrm{a}$ reflux condenser under nitrogen. Dry DMF $(180 \mathrm{~mL})$ was added, followed by the dropwise addition of dry, redistilled triethylamine $(3.6 \mathrm{~mL})$. The reaction mixture was stirred at $63{ }^{\circ} \mathrm{C}$ for 8.5 hours, at which time it was allowed to cool to room temperature and the solvent was removed by rotary evaporation. The resulting viscous oil was sonicated for 5 minutes in $25 \mathrm{~mL} \mathrm{MeOH}$ and filtered, washing with $20 \mathrm{~mL} \mathrm{MeOH}$. The solid residue was suspended in $25 \mathrm{~mL}$ DCM, sonicated for 5 minutes, and filtered, washing with $25 \mathrm{~mL}$ DCM. The residual solid (comprised mostly of octanitro cavitand) was set aside, and the filtrate was concentrated by rotary evaporation yielding a solid. Flash chromatography (DCM, then 2\% EtOAc in DCM) followed by rotary evaporation gave the hexanitro cavitand as a yellow solid (665 mg, 15\% yield). ${ }^{1} \mathrm{H}$ NMR $\left(\mathrm{CD}_{2} \mathrm{Cl}_{2}, 600 \mathrm{MHz}\right) \delta 7.70(\mathrm{~s}, 2 \mathrm{H}), 7.68(\mathrm{~s}, 2 \mathrm{H}), 7.66(\mathrm{~s}, 2 \mathrm{H}), 6.95(\mathrm{~s}, 2 \mathrm{H}), 6.86(\mathrm{br} \mathrm{s}, 2 \mathrm{H})$, 6.72 (br s, 2H), 5.96 (br s, 2H), 4.22 (t, $J=7.7 \mathrm{~Hz}, 1 \mathrm{H}, \operatorname{ArCHRAr}), 3.89$ (t, $J=7.6 \mathrm{~Hz}, 3 \mathrm{H}$, ArCHRAr). 2.09 (m, 8H, $\mathrm{RCH}_{2} \mathrm{CH}_{3}$ ), 0.89 (m, 12H, $\mathrm{RCH}_{2} \mathrm{CH}_{3}$ ). MS (MALDI-FTMS: [M-H] ${ }^{-}$) calcd. for $\mathrm{C}_{54} \mathrm{H}_{39} \mathrm{~N}_{6} \mathrm{O}_{20}$ 1091.2225, found 1091.2224.

\footnotetext{
${ }^{i}$ Pangborn, A. B.; Giardello, M. A.; Grubbs, R. H.; Rosen, R. K.; Timmers, F. J. Organometallics 1996, 15, 1518.

ii Purse, B. W.; Shivanyuk, A.; Rebek, J., Jr. Chem. Commun. 2002, 2612 and references therein.
} 
Scheme S1. Synthesis of introverted acid cavitand 1a.
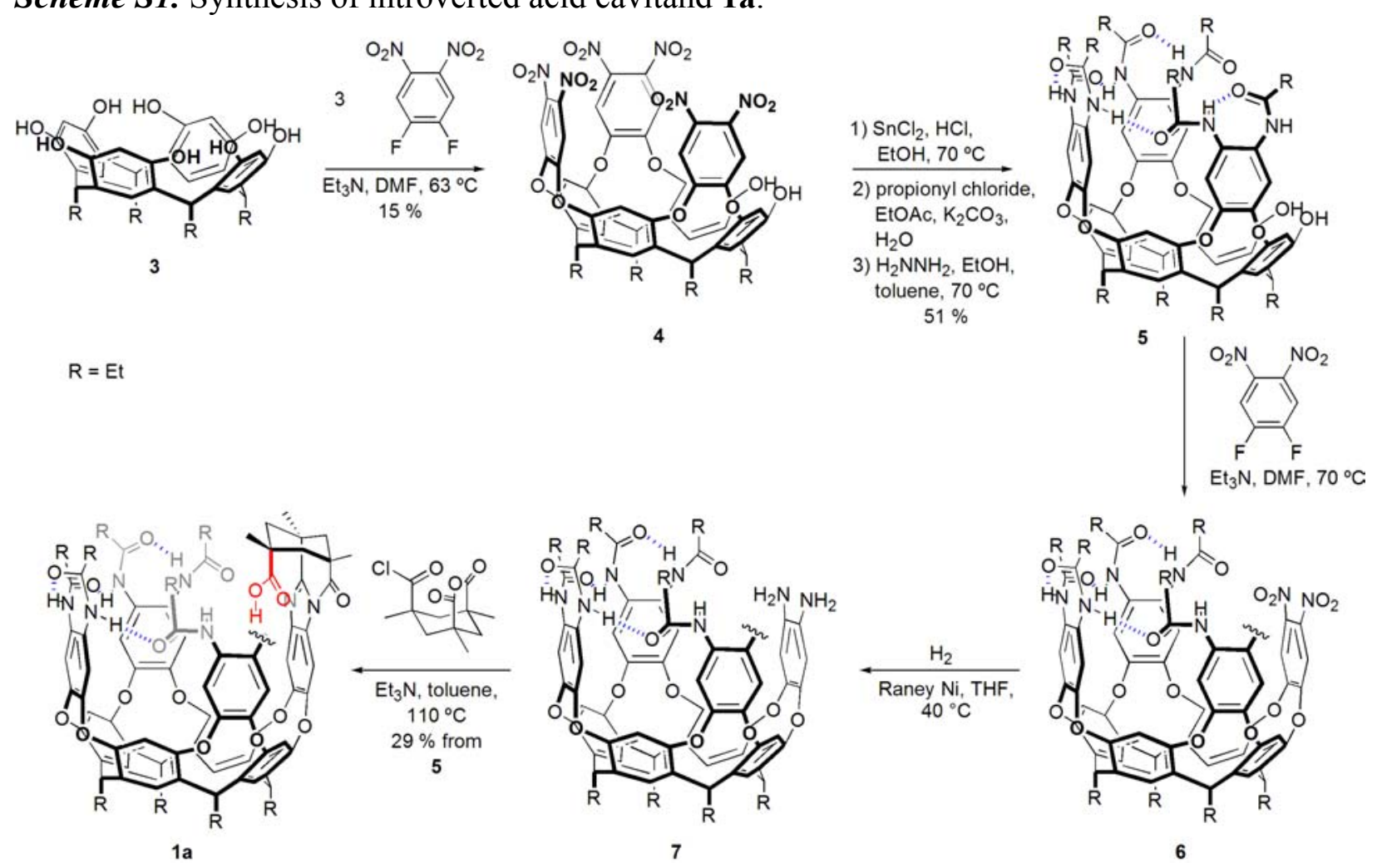

Hexaamide Cavitand 5. Hexanitro cavitand 4 (572 $\mathrm{mg}, 0.523 \mathrm{mmol})$ was suspended in 4:1 EtOH:37 \% HCl in a $100 \mathrm{~mL}$ 3-necked flask under $\mathrm{N}_{2}$. $\mathrm{SnCl}_{2}$ (anhydrous, $2.5 \mathrm{~g}, 13 \mathrm{mmol}$ ) was added, and the reaction mixture was heated at $70{ }^{\circ} \mathrm{C}$ for 10 hours with stirring. The color changed from yellow to orange to pale yellow as the reaction reached completion. Rotary evaporation removed the solvent, and the pale yellow material was transferred to a beaker. EtOAc $(50 \mathrm{~mL})$ was added, followed by the slow addition of a solution of $\mathrm{K}_{2} \mathrm{CO}_{3}(8.5 \mathrm{~g})$ in water $(40 \mathrm{~mL})$. More EtOAc $(50 \mathrm{~mL})$ was added, stirring was made vigorous, and propionyl chloride $(2 \times 650 \mu \mathrm{L})$ was added, waiting 30 min between additions. The organic layer was separated and combined with two EtOAc extractions $(50 \mathrm{~mL}$ each) of the aqueous layer. The EtOAc solution was washed with brine, dried over anhydrous $\mathrm{Na}_{2} \mathrm{SO}_{4}$, and rotary evaporated to remove the solvent. This crude hexaamide diester was dissolved in 1:1 EtOH:toluene $(30 \mathrm{~mL})$ under $\mathrm{N}_{2}$. Hydrazine hydrate $(250 \mu \mathrm{L})$ was added, and the reaction mixture was heated at $70{ }^{\circ} \mathrm{C}$ with stirring for 1 hour. The reaction mixture was allowed to cool, and the solvent removed by rotary evaporation. DCM $(5 \mathrm{~mL})$ was added, and the solution was allowed to rest at room temperature for 12 hours, during which time a white precipitate formed. DCM $(5 \mathrm{~mL})$ was again added, and the precipitate was collected by filtration through a frit and retained. The filtrate was concentrated by rotary evaporation and purified by flash chromatography $(1 \% \mathrm{MeOH}$ in DCM to $1.25 \% \mathrm{MeOH}$ in DCM) to give hexaamide 5 as a clear oil. This was combined with the precipitate, yielding $333 \mathrm{mg}(51 \%) . R_{\mathrm{f}}=0.19\left(3 \% \mathrm{MeOH}\right.$ in DCM). ${ }^{1} \mathrm{H}$ NMR (acetone- $d_{6}, 600$ MHz) $\delta 9.54$ (br s, 2H, NH), 9.41 (br s, 2H, NH), 9.17 (br s, 2H, OH), 8.95 (br s, 2H, NH), 7.89 $(\mathrm{s}, 2 \mathrm{H}), 7.75(\mathrm{~s}, 2 \mathrm{H}), 7.71(\mathrm{~s}, 2 \mathrm{H}), 7.67(\mathrm{~s}, 4 \mathrm{H}), 7.51(\mathrm{~s}, 2 \mathrm{H}), 6.76(\mathrm{~s}, 2 \mathrm{H}), 5.73(\mathrm{t}, J=8.1 \mathrm{~Hz}, 1 \mathrm{H}$, ArCHRAr), 5.61 (m, 2H, ArCHRAr), 4.25 (t, $J=8.0,1 \mathrm{H}, \operatorname{ArCHRAr}), 2.35(\mathrm{~m}, 20 \mathrm{H}), 1.10$ (m, 30H). MS (MALDI-FTMS: $\mathrm{MNa}^{+}$) calcd. for $\mathrm{C}_{72} \mathrm{H}_{76} \mathrm{~N}_{6} \mathrm{O}_{14} \mathrm{Na}^{+} 1271.5311$, found 1271.5326 . 
Introverted Acid Cavitand 1a. Hexaamide cavitand 5 (515 mg, $0.412 \mathrm{mmol}$ ) and 1,2-difluoro4,5-dinitrobenzene (92 mg, $0.45 \mathrm{mmol}$ ) were dissolved in dry DMF ( $80 \mathrm{~mL})$ under $\mathrm{N}_{2}$. Dry redistilled triethylamine $(188 \mu \mathrm{L}, 1.35 \mathrm{mmol})$ was added dropwise and the reaction mixture was stirred at $70{ }^{\circ} \mathrm{C}$ for 6 hours. The solvent was removed by rotary evaporation, and the concentrated material was dried in vacuo. The concentrate was then dissolved in THF. Raney nickel was added, and the solution reaction mixture was stirred at $40{ }^{\circ} \mathrm{C}$ under $\mathrm{H}_{2}(1 \mathrm{~atm})$ for 4 hours until the color had changed from yellow to pale yellow / colorless and was complete by TLC. The mixture was filtered through celite, washing with THF, and rotary evaporated to give the crude diamine, which was dried in vacuo in the dark. Kemp's triacid chloride anhydride ${ }^{\text {iii }}$ (166 mg, $0.642 \mathrm{mmol}$ ), toluene (anhydrous, $13.3 \mathrm{~mL}$ ), and freshly redistilled triethylamine (332 $\mu \mathrm{L}, 2.38 \mathrm{mmol}$ ) were added to the crude diamine. The reaction mixture was stirred for $30 \mathrm{~min}$. at room temperature under $\mathrm{N}_{2}$, then heated at reflux for 6 hours, after which it was allowed to cool to room temperature and left to stir for 12 hours. The reaction mixture was then filtered through celite, washing with DCM. The solvent was removed by rotary evaporation, and the product was redissolved in DCM $(65 \mathrm{~mL})$. The solution was washed with $10 \% \mathrm{HCl}(3 \mathrm{x})$, brine, and dried over $\mathrm{Na}_{2} \mathrm{SO}_{4}$. Rotary evaporation gave the crude product as a viscous oil. Flash chromatography (3:1 EtOAc:DCM) gave the pure introverted acid 1 as a white solid (188 $\mathrm{mg}, 29 \%$ yield) which could be recrystallized from chloroform / hexanes. $R_{\mathrm{f}}=0.29$ (EtOAc). ${ }^{1} \mathrm{H} \mathrm{NMR}\left(\mathrm{CDCl}_{3}, 600\right.$ MHz) $\delta 9.85(\mathrm{~s}, 1 \mathrm{H}), 9.42(\mathrm{~s}, 1 \mathrm{H}), 9.09(\mathrm{~s}, 1 \mathrm{H}), 8.57(\mathrm{~s}, 1 \mathrm{H}), 8.25(\mathrm{~s}, 1 \mathrm{H}), 8.10$ (br s, 1H), 8.06 $(\mathrm{s}, 1 \mathrm{H}), 7.83(\mathrm{~s}, 1 \mathrm{H}), 7.80(\mathrm{~s}, 1 \mathrm{H}), 7.65(\mathrm{~s}, 1 \mathrm{H}), 7.47(\mathrm{~s}, 1 \mathrm{H}), 7.26(\mathrm{~m}, 10 \mathrm{H}), 7.10(\mathrm{~s}, 1 \mathrm{H}), 5.75(\mathrm{t}$, $J=9,1 \mathrm{H}), 5.70(\mathrm{t}, J=9,1 \mathrm{H}), 5.66(\mathrm{t}, J=9,1 \mathrm{H}), 5.59(\mathrm{t}, J=9,1 \mathrm{H}), 2.78(\mathrm{~d}, J=14,1 \mathrm{H}), 2.72$ $(\mathrm{d}, J=14,1 \mathrm{H}), 2.35(\mathrm{~m}, 20 \mathrm{H}), 2.00(\mathrm{~m}, 2 \mathrm{H}), 1.61(\mathrm{~s}, 3 \mathrm{H}), 1.44(\mathrm{~s}, 3 \mathrm{H}), 1.24(\mathrm{~m}, 16 \mathrm{H}), 1.19(\mathrm{~s}$, $3 \mathrm{H}), 1.07(\mathrm{~m}, 6 \mathrm{H}), 1.00(\mathrm{t}, J=7,3 \mathrm{H}), 0.97(\mathrm{t}, J=7,3 \mathrm{H}), 0.90(\mathrm{t}, J=7,3 \mathrm{H})$. IR (thin film; $\left.\mathrm{cm}^{-1}\right)$ 3252 (br), 2966, 2935, 2876, 1731, 1667, 1600, 1513, 1486, 1403, 1328, 1276, 1182, 1154, 1121, 1055, 1024, 938, 895, 735, 668. MS (MALDI-FTMS: $\mathrm{MH}^{+}$) calcd. for $\mathrm{C}_{90} \mathrm{H}_{92} \mathrm{~N}_{8} \mathrm{O}_{17} \mathrm{H}^{+}$ 1557.6653, found 1557.6686.

\footnotetext{
iii Rebek, J., Jr.; Marshall, L.; Wolak, R.; Parris, K.; Killoran, M.; Askew, B.; Nemeth, D.; Islam, N. J. Am. Chem.
} Soc. 1985, 107, 7446. 
Figure S1. ${ }^{1} \mathrm{H}$ NMR spectrum of $1 \mathrm{a}$ at $300 \mathrm{~K}$ in $\mathrm{CDCl}_{3}$.

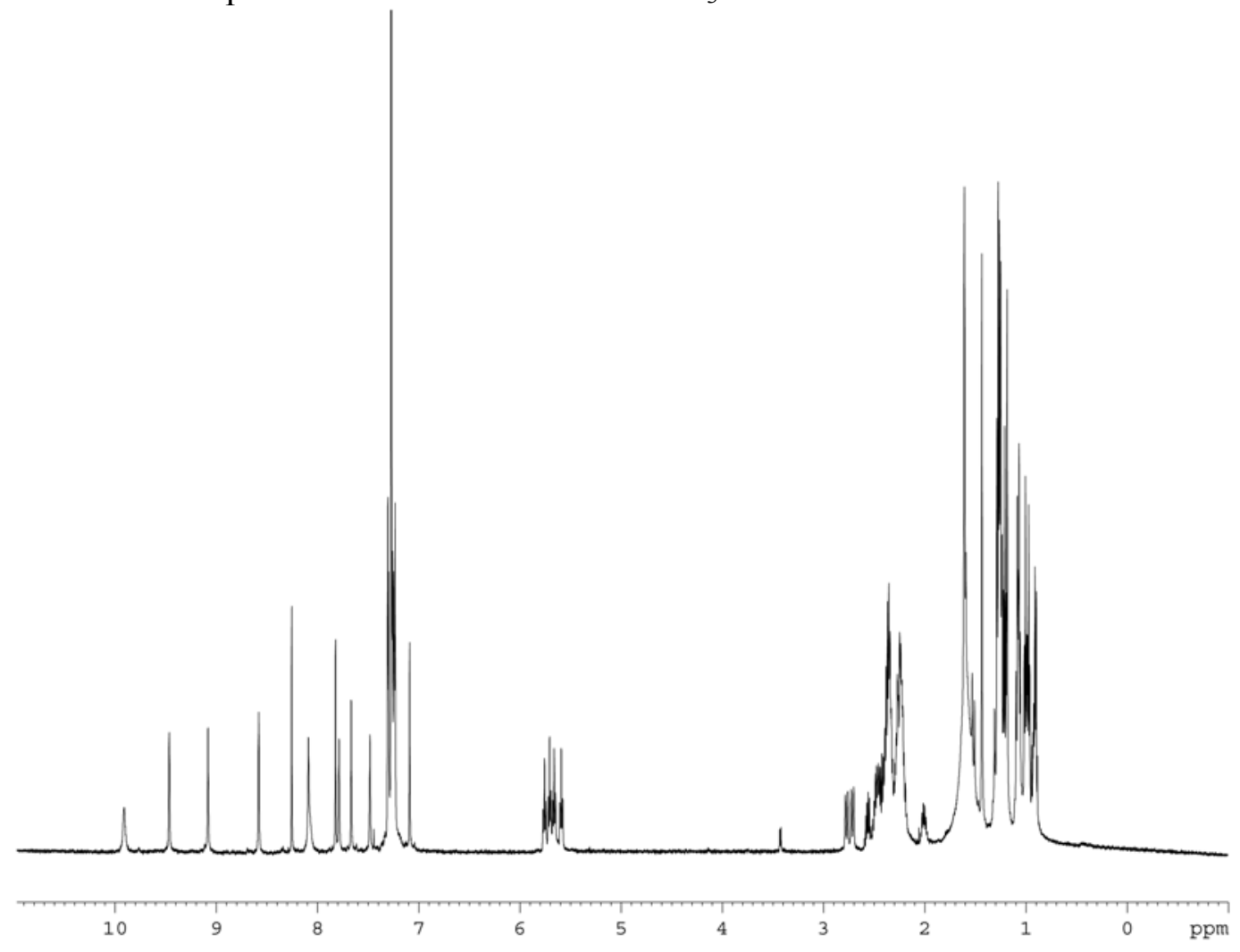

Introverted Methyl Ester 1b. Introverted acid cavitand 1a $(19.2 \mathrm{mg}, 0.0123 \mathrm{mmol})$ was dissolved in chloroform- $d(700 \mu \mathrm{L})$ in an NMR tube. Diazomethane (freshly prepared, solution in $\mathrm{Et}_{2} \mathrm{O}$ ) was added dropwise until the yellow color remained. A ${ }^{1} \mathrm{H}$ NMR spectrum immediately acquired showed quantitative transformation to the methyl ester (reaction time $<3$ minutes). $R_{\mathrm{f}}=$ 0.36 (4:1 EtOAc:DCM). ${ }^{1} \mathrm{H}$ NMR $\left(\mathrm{CDCl}_{3}, 600 \mathrm{MHz}\right) \delta 9.84(\mathrm{~s}, 1 \mathrm{H}), 9.55(\mathrm{~s}, 1 \mathrm{H}), 9.15(\mathrm{~s}, 1 \mathrm{H})$, $8.69(\mathrm{~s}, 1 \mathrm{H}), 8.60(\mathrm{~s}, 1 \mathrm{H}), 8.32(\mathrm{~s}, 1 \mathrm{H}), 8.25(\mathrm{~s}, 1 \mathrm{H}), 8.18(\mathrm{~s}, 1 \mathrm{H}), 8.03(\mathrm{~s}, 1 \mathrm{H}), 7.78(\mathrm{~s}, 1 \mathrm{H}), 7.65$ $(\mathrm{s}, 1 \mathrm{H}), 7.44(\mathrm{~s}, 1 \mathrm{H}), 7.37(\mathrm{~s}, 1 \mathrm{H}), 7.36(\mathrm{~s}, 1 \mathrm{H}), 7.34(\mathrm{~s}, 1 \mathrm{H}), 7.31(\mathrm{~s}, 1 \mathrm{H}), 7.27(\mathrm{~s}, 1 \mathrm{H}), 7.25(\mathrm{~s}$, $1 \mathrm{H}), 7.24(\mathrm{~s}, 1 \mathrm{H}), 7.22(\mathrm{~s}, 1 \mathrm{H}), 7.11(\mathrm{~s}, 1 \mathrm{H}), 7.06(\mathrm{~s}, 1 \mathrm{H}), 5.78(\mathrm{t}, J=8,1 \mathrm{H}), 5.72(\mathrm{t}, J=8,2 \mathrm{H})$, $5.66(\mathrm{t}, J=8,1 \mathrm{H}), 2.75(\mathrm{~d}, J=14,1 \mathrm{H}), 2.61(\mathrm{~d}, J=14,1 \mathrm{H}), 2.39(\mathrm{~m}, 22 \mathrm{H}), 1.94(\mathrm{~s}, 3 \mathrm{H}$, $\left.\mathrm{CO}_{2} \mathrm{CH}_{3}\right), 1.62(\mathrm{~s}, 3 \mathrm{H}), 1.42(\mathrm{~s}, 3 \mathrm{H}), 1.26(\mathrm{~s}, 3 \mathrm{H}), 1.25(\mathrm{~m}, 20 \mathrm{H}), 1.04(\mathrm{~m}, 12 \mathrm{H})$. IR (thin film; $\left.\mathrm{cm}^{-1}\right) 3251$ (br), 2963, 2917, 2849, 1726, 1666, 1599, 1513, 1485, 1462, 1402, 1383, 1316, 1266, 1182, 1153, 1121, 938, 895, 737, 703. MS (MALDI-FTMS: $\mathrm{MNa}^{+}$) calcd. for $\mathrm{C}_{91} \mathrm{H}_{94} \mathrm{~N}_{8} \mathrm{O}_{17} \mathrm{Na}^{+}$ 1593.6629, found 1593.6669 . 
Figure S2. ${ }^{1} \mathrm{H}$ NMR spectrum of $\mathbf{1 b}$ at $300 \mathrm{~K}$ in $\mathrm{CDCl}_{3}$.

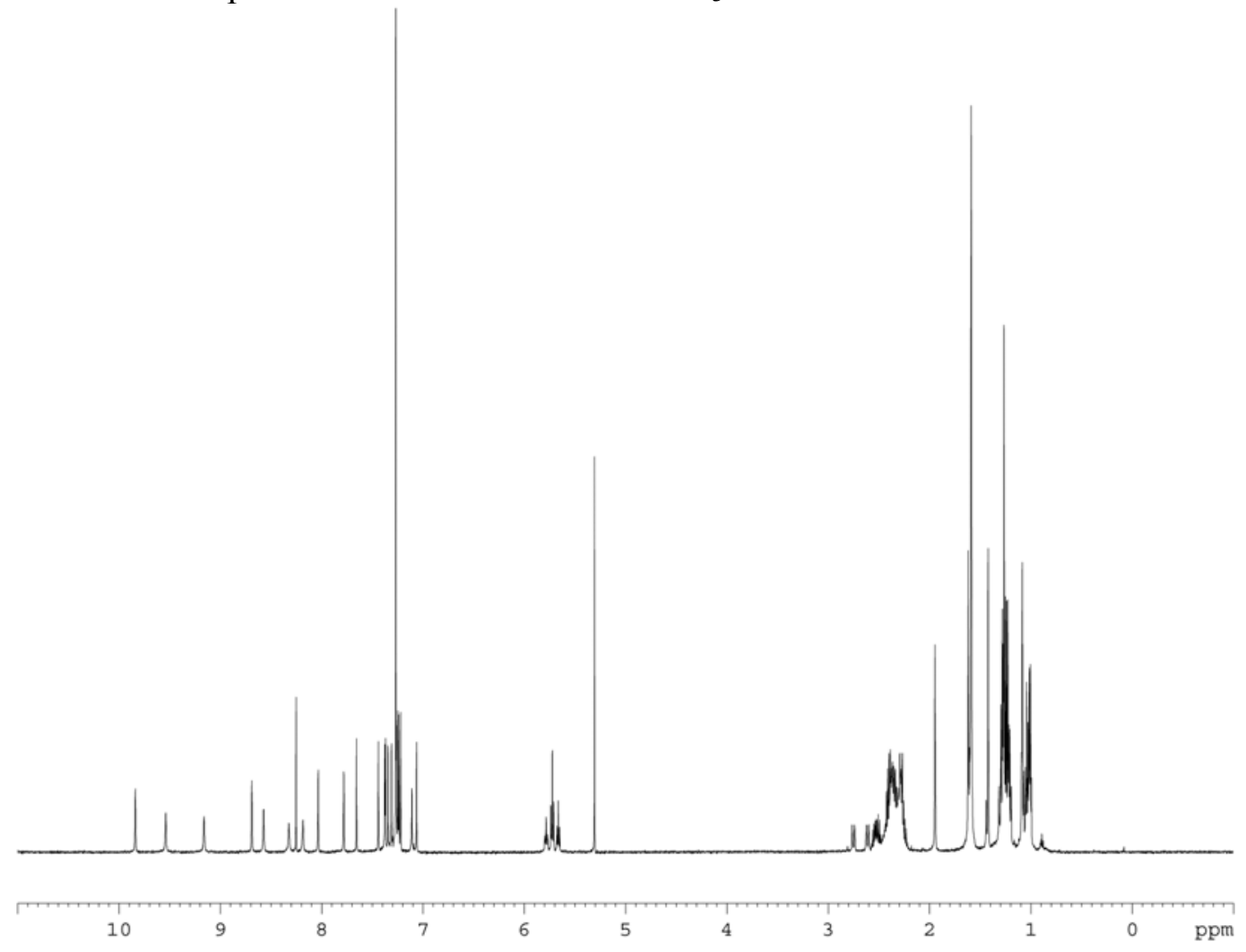

Introverted Sodium Salt 1c. Introverted acid cavitand 1a $(5.0 \mathrm{mg}, 3.2 \mu \mathrm{mol})$ was dissolved in DCM $(2 \mathrm{~mL})$ and mixed vigorously with $1 \mathrm{M} \mathrm{NaOH}(2 \mathrm{~mL})$ by shaking for 10 minutes. The layers were separated, and the organic layer was dried over anhydrous $\mathrm{Na}_{2} \mathrm{SO}_{4}$. Rotary evaporation gave 1c as an off-white solid (5.1 mg, $100 \%$ yield). ${ }^{1} \mathrm{H} \mathrm{NMR}\left(\mathrm{CDCl}_{3}, 600 \mathrm{MHz}\right) \delta$ $9.98(\mathrm{~s}, 1 \mathrm{H}), 9.88(\mathrm{~s}, 1 \mathrm{H}), 9.59(\mathrm{~s}, 1 \mathrm{H}), 9.12(\mathrm{~s}, 1 \mathrm{H}), 8.63(\mathrm{~s}, 1 \mathrm{H}), 8.58(\mathrm{~s}, 1 \mathrm{H}), 8.49(\mathrm{~s}, 1 \mathrm{H}), 7.87$ (s, 1H), $7.68(\mathrm{~s}, 1 \mathrm{H}), 7.65(\mathrm{~s}, 1 \mathrm{H}), 7.39(\mathrm{~s}, 1 \mathrm{H}), 7.35(\mathrm{~s}, 2 \mathrm{H}), 7.34(\mathrm{~s}, 1 \mathrm{H}), 7.26(\mathrm{~m}, 7 \mathrm{H}), 7.05(\mathrm{~s}$, $1 \mathrm{H}), 5.81(\mathrm{t}, J=8,1 \mathrm{H}), 5.76(\mathrm{t}, J=9,1 \mathrm{H}), 5.74(\mathrm{t}, J=9,1 \mathrm{H}), 5.66(\mathrm{t}, J=9,1 \mathrm{H}), 2.89(\mathrm{~d}, J=13$, $1 \mathrm{H}), 2.84(\mathrm{~d}, J=13,1 \mathrm{H}), 2.34(\mathrm{~m}, 16 \mathrm{H}), 1.54(\mathrm{~s}, 3 \mathrm{H}), 1.42(\mathrm{~s}, 3 \mathrm{H}), 1.26(\mathrm{~m}, 8 \mathrm{H}), 1.22(\mathrm{~s}, 3 \mathrm{H})$, $1.08(\mathrm{t}, J=7,6 \mathrm{H}), 1.05(\mathrm{t}, J=7,6 \mathrm{H}), 1.00(\mathrm{t}, J=7,6 \mathrm{H}), 0.90(\mathrm{t}, J=7,12 \mathrm{H})$. IR (thin film; $\mathrm{cm}^{-}$ $\left.{ }^{1}\right) 3251$ (br), 2963, 2931, 2875, 1725, 1667, 1599, 1513, 1485, 1462, 1403, 1327, 1277, 1221, $1182,1153,1121,1057,1025,937,894,736$.

Model acid 2a. 1,2-Dinitro-4,5-bis-octyloxy-benzene (300 $\mathrm{mg}, 0.71 \mathrm{mmol})$ was dissolved in toluene and a catalytic amount of Raney Nickel was added. The mixture was stirred under $\mathrm{H}_{2}(1$ atm) at $40^{\circ} \mathrm{C}$ until complete by TLC (9:1 DCM:MeOH), approximately 2 hours. The supernatant solution was decanted from the Raney Nickel, and Kemp's triacid chloride anhydride $\mathrm{iii}^{\mathrm{iii}}(171 \mathrm{mg}$, $0.71 \mathrm{mmol})$ was added, followed by the dropwise addition of triethylamine $(0.6 \mathrm{~mL})$. The reaction mixture was heated at reflux for 12 hours. Removal of the solvent by rotary evaporation, followed by flash chromatography $(1.5 \% \mathrm{MeOH}$ in DCM) yielded the product as an off-white solid $\left(75 \mathrm{mg}, 19 \%\right.$ yield). ${ }^{1} \mathrm{H}$ NMR $\left(\mathrm{CDCl}_{3}, 600 \mathrm{MHz}\right) \delta 7.61(\mathrm{~s}, 1 \mathrm{H}), 7.09(\mathrm{~s}, 1 \mathrm{H}), 3.99(\mathrm{~m}$, $2 \mathrm{H}), 3.92(\mathrm{~m}, 2 \mathrm{H}), 2.61(\mathrm{~d}, J=13,1 \mathrm{H}), 2.59(\mathrm{~d}, J=13,1 \mathrm{H}), 2.17(\mathrm{~d}, J=13,1 \mathrm{H}), 1.81(\mathrm{~m}, 4 \mathrm{H})$, $1.52(\mathrm{~s}, 3 \mathrm{H}), 1.52(\mathrm{~d}, J=13,1 \mathrm{H}), 1.46(\mathrm{~m}, 4 \mathrm{H}), 1.35(\mathrm{~s}, 3 \mathrm{H}), 1.32(\mathrm{~m}, 17 \mathrm{H}), 1.21(\mathrm{~d}, J=14,1 \mathrm{H})$, 
$1.04(\mathrm{~s}, 3 \mathrm{H}), 0.91(\mathrm{~m}, 6 \mathrm{H}) .{ }^{13} \mathrm{C} \mathrm{NMR}\left(\mathrm{CDCl}_{3}, 150 \mathrm{MHz}\right) \delta 173.27,157.02,147.69,147.61$, 136.59, 124.22, 103.43, 99.88m 69.29, 69.24, 47.19, 46.93, 44.49, 41.89, 41.55, 34.80, 32.07, $30.35,29.69,29.65,29.55,29.48,29.39,26.41,26.36,25.91,24.95,22.92,14.35$. IR (thin film; $\mathrm{cm}^{-1}$ ) 3590-2210 (br, $\left.v_{\mathrm{CO}_{2} \mathrm{H}}\right), 2926,2855,1732,1548,1487,1468,1426,1381,1369,1325,1294$, 1240, 1172, 1112, 999, 873, 845, 785, 754, 723, 638. MS (MALDI-FTMS: $\mathrm{MH}^{+}$) calcd. for $\mathrm{C}_{34} \mathrm{H}_{52} \mathrm{~N}_{2} \mathrm{O}_{5} \mathrm{H}^{+}$569.3949, found 569.3941.

Model methyl ester 2b. Model acid 2a (27 mg, $0.047 \mathrm{mmol})$ was dissolved in DCM (5 mL). An ethereal solution of diazomethane was added until the yellow color persisted. Rotary evaporation followed by flash chromatography (3:1 hexanes:EtOAc) yielded the product as a clear oil (19 mg, $69 \%$ yield). ${ }^{1} \mathrm{H}$ NMR $\left(\mathrm{CDCl}_{3}, 600 \mathrm{MHz}\right) \delta 7.77(\mathrm{~s}, 1 \mathrm{H}), 7.22(\mathrm{~s}, 1 \mathrm{H}), 4.04(\mathrm{~m}, 4 \mathrm{H}), 2.82(\mathrm{~d}$, $J=14,1 \mathrm{H}), 2.79\left(\mathrm{~s}, 3 \mathrm{H},-\mathrm{CO}_{2} \mathrm{CH}_{3}\right), 2.77(\mathrm{~d}, J=14,1 \mathrm{H}), 2.22(\mathrm{~d}, J=13,1 \mathrm{H}), 1.84(\mathrm{~m}, 4 \mathrm{H}), 1.59$ $(\mathrm{s}, 3 \mathrm{H}), 1.57(\mathrm{~d}, J=14,1 \mathrm{H}), 1.48(\mathrm{~m}, 4 \mathrm{H}), 1.44(\mathrm{~d}, J=13,1 \mathrm{H}), 1.40(\mathrm{~s}, 3 \mathrm{H}), 1.31(\mathrm{~m}, 17 \mathrm{H}), 1.18$ $(\mathrm{s}, 3 \mathrm{H}), 0.89(\mathrm{t}, J=7,6 \mathrm{H}) .{ }^{13} \mathrm{C} \mathrm{NMR}\left(\mathrm{CDCl}_{3}, 150 \mathrm{MHz}\right) \delta 175.13,173.51,157.71,148.14$, 124.90, 104.07, 100.63, 69.90, 69.75, 52.18, 47.28, 46.73, 45.02, 42.33, 41.74, 34.93, 32.05, $30.47,29.64,29.49,29.47,29.43,26.29,26.24,26.08,25.07,22.89,14.32$. IR (thin film; $\mathrm{cm}^{-1}$ ) 2926, 2855, 1732, 1553, 1465, 1383, 1305, 1197, 1172, 1109, 995, 962, 854, 756. MS (MALDIFTMS: $\mathrm{MH}^{+}$) calcd. for $\mathrm{C}_{35} \mathrm{H}_{54} \mathrm{~N}_{2} \mathrm{O}_{5} \mathrm{H}^{+}$583.4105, found 583.4097.

Model sodium salt 2c. Model acid 2a $(5.0 \mathrm{mg}, 8.7 \mu \mathrm{mol})$ was dissolved in DCM (2 mL) and mixed vigorously with $1 \mathrm{M} \mathrm{NaOH}(2 \mathrm{~mL})$ by shaking for 10 minutes. The layers were separated, and the organic layer was dried over anhydrous $\mathrm{Na}_{2} \mathrm{SO}_{4}$. Rotary evaporation gave $1 \mathrm{c}$ as an offwhite solid (5.2 mg, $100 \%$ yield). ${ }^{1} \mathrm{H}$ NMR $\left(\mathrm{CDCl}_{3}, 600 \mathrm{MHz}\right) \delta 7.61(\mathrm{~s}, 1 \mathrm{H}), 7.10(\mathrm{~s}, 1 \mathrm{H}), 3.97$ $(\mathrm{m}, 4 \mathrm{H}), 2.67(\mathrm{~d}, J=13,2 \mathrm{H}), 2.11(\mathrm{~d}, J=13,1 \mathrm{H}), 1.78(\mathrm{~m}, 4 \mathrm{H}), 1.50(\mathrm{~s}, 3 \mathrm{H}), 1.47(\mathrm{~m}, 5 \mathrm{H}), 1.33$ $(\mathrm{s}, 3 \mathrm{H}), 1.31(\mathrm{~m}, 17 \mathrm{H}), 1.13(\mathrm{~d}, J=13,1 \mathrm{H}), 1.01(\mathrm{~s}, 3 \mathrm{H}), 0.90(\mathrm{t}, J=7,3 \mathrm{H})$. IR (thin film; $\left.\mathrm{cm}^{-1}\right)$ 3703-2574 (br, $\mathrm{H}_{2} \mathrm{O}$ ), 2926, 2855, 1724, 1568, 1467, 1423, 1396, 1381, 1364, 1316, 1292, 1220 , 1209, 1161, 1110, 996, 967, 860, 757, 722, 637. 


\section{Sample Caviplex Spectrum}

Figure S3. Stack plot showing the reaction between quinuclidine and $\mathbf{1 b}$ after 5 min compared to $N$-methylquinuclidinium@1c prepared as a standard.

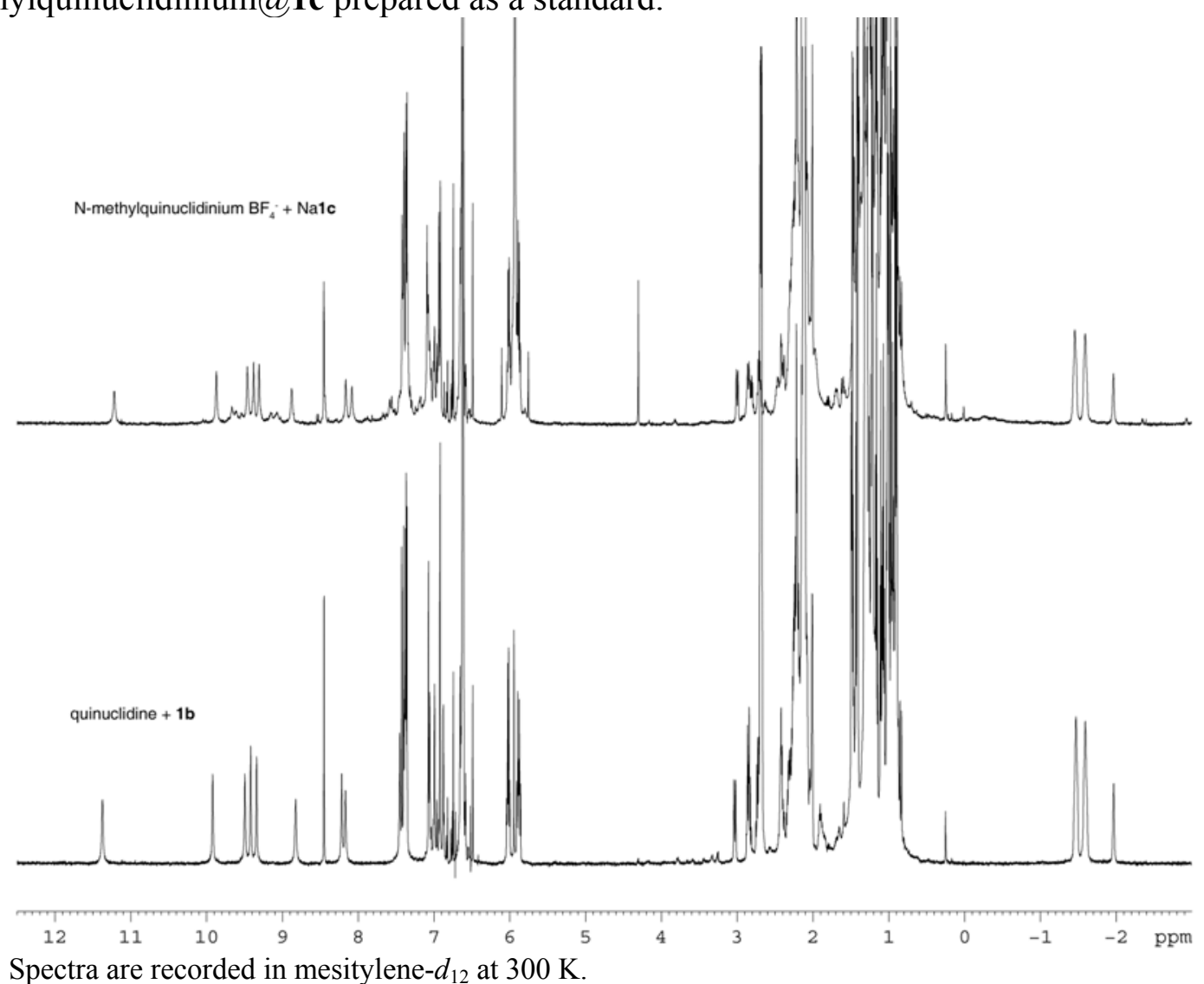




\section{Competitive Binding Experiments}

We measured relative association constants $K_{\text {rel }}$ of amines and methyl ester cavitand $\mathbf{1 b}$ in order to estimate the intrinsic reactivity of each respective complex. We first attempted to measure association constants for slowly-reacting amines by titration of guest amine into a solution of host under reaction conditions. ${ }^{\text {iv }}$ Under these condition of fast exchange, little structural information is provided and it was impossible to differentiation true host-guest complexation from other modes of association, for example, hydrogen bonding. To allay these concerns, we chose to measure association constants by competitive binding experiments at low temperature. ${ }^{\mathrm{V}}$ We found that it was possible to observe a complex between amine and methyl ester at $240 \mathrm{~K}$ by ${ }^{1} \mathrm{H}$ NMR and that integration could be used to measure relative association constants. The relative association constants in Table 1 were determined by pairwise competition binding experiments of guest amines with $N$-methylmorpholine using the following equation:

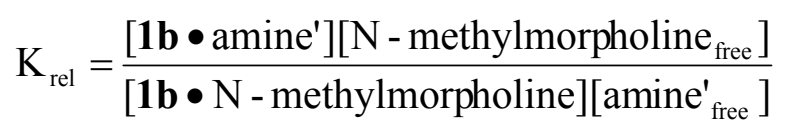

Sample spectrum used for $\mathrm{K}_{\text {rel }}$ determination (in mesitylene- $d_{12}$ at $240 \mathrm{~K}$ ):

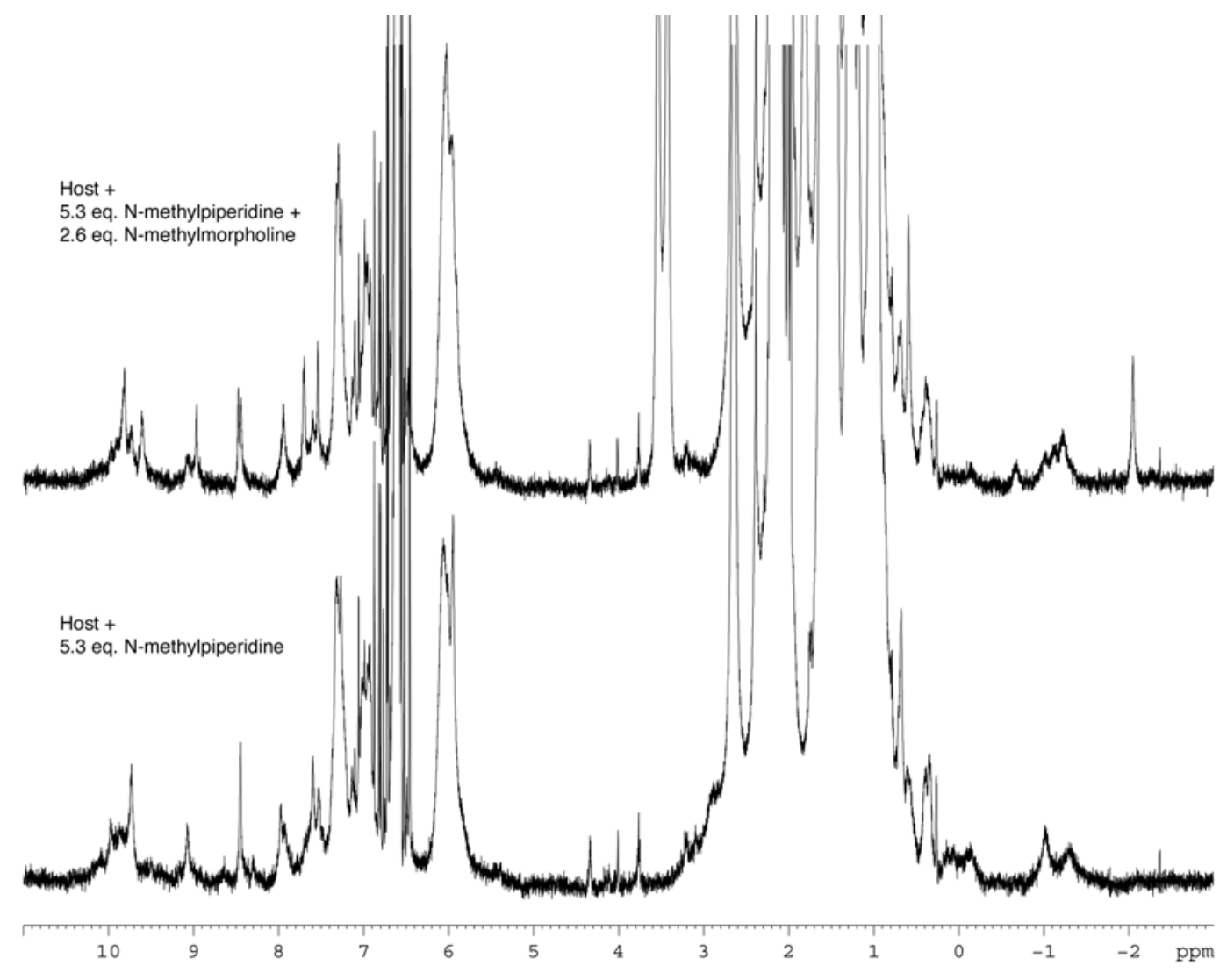

\footnotetext{
${ }^{i v}$ See: Connors, K. A. Binding Constants Wiley \& Sons: New York, 1987; Chapter 5.

${ }^{v}$ Hof, F. A. Self-Assembled Tetrameric Capsules. Ph.D. Thesis, The Scripps Research Institute, La Jolla, CA, March 2003.
} 


\section{Kinetic Study Data}

In order to ascertain the transition state theory activation parameters for the reaction of $\mathbf{1 b}$ with 2-(dimethylamino)ethanol, a kinetic study was undertaken. We chose to investigate this amine because its intermediate rate of reaction presented the fewest technical difficulties in making kinetic measurements. The rate of quaternization of 2-(dimethylamino)ethanol was measured at five temperatures: $35,40,45,50$, and $55^{\circ} \mathrm{C}$. In a typical experiment, an accurately prepared solution of amine $(5.7 \mu \mathrm{mol})$ was added to a solution of methyl ester cavitand $(\sim 2 \mathrm{mg}$, $1.3 \mu \mathrm{mol})$ to make a total volume of $600 \mu \mathrm{L}$ mesitylene- $d_{12}$ in an NMR tube. Setting a delay time equal to five times T1, the integral area of the amine peak could be used to calculate the exact concentration of $\mathbf{1 b}$. Immediately upon mixing, the sample was placed in the NMR at the desired temperature and the reaction monitored by periodic spectral acquisition. Second order rate law plots at each temperature resulted in straight lines (1st order in each reactant did not), and the rate constant at each temperature was determined. An Eyring plot gave a straight line, from which the activation parameters $\Delta \mathrm{H}^{*}=25.1 \pm 0.5 \mathrm{kcal} \mathrm{mol}^{-1}$ and $\Delta \mathrm{S}^{*}=12 \pm 2 \mathrm{cal} \mathrm{mol}^{-1} \mathrm{~K}^{-1}$ were calculated.

$35^{\circ} \mathrm{C}$

\begin{tabular}{|c|c|c|c|c|c|c|c|}
\hline & $\begin{array}{l}{[\mathrm{Me}]_{0}} \\
{[\text { amine }]_{0}}\end{array}$ & $\begin{array}{l}9.60 \mathrm{E}-04 \\
9.50 \mathrm{E}-03\end{array}$ & & & & & \\
\hline Time / s & [Me ester] / M & [amine] / M & [pdt] / M & $\ln \left(\left([\text { Me ester }]_{0}-\mathrm{x}\right) /\left(\left([\text { amine }]_{0}-\mathrm{x}\right)\right)\right.$ & $-3.35211 \mathrm{E}-05$ & -2.448291 & \\
\hline 840 & 8.93E-04 & 9.43E-03 & $6.72 \mathrm{E}-05$ & -2.357585665 & 2.02864E-06 & 0.045975 & \\
\hline 1620 & 7.58E-04 & $9.30 \mathrm{E}-03$ & 2.02E-04 & -2.50638667 & 0.934940307 & 0.10776 & \\
\hline 3900 & 7.87E-04 & $9.33 \mathrm{E}-03$ & 1.73E-04 & -2.472207796 & 273.0394964 & 19 & \\
\hline 6180 & 5.57E-04 & $9.10 \mathrm{E}-03$ & 4.03E-04 & -2.793471873 & 3.170600118 & 0.220633 & \\
\hline 8460 & 5.76E-04 & $9.12 \mathrm{E}-03$ & 3.84E-04 & -2.76167873 & & & \\
\hline 9960 & 5.09E-04 & $9.05 \mathrm{E}-03$ & $4.51 \mathrm{E}-04$ & -2.878332419 & & & $+/-$ \\
\hline 11460 & 5.09E-04 & $9.05 E-03$ & 4.51E-04 & -2.878332419 & $\mathrm{~m}$ & $-3.35 E-05$ & 2.03E-06 \\
\hline 13740 & $4.51 \mathrm{E}-04$ & 8.99E-03 & 5.09E-04 & -2.9920909 & $\mathrm{~b}$ & -2.448291 & 0.045975 \\
\hline 16020 & 4.61E-04 & $9.00 \mathrm{E}-03$ & 4.99E-04 & -2.972104632 & & & \\
\hline 17580 & 4.51E-04 & 8.99E-03 & 5.09E-04 & -2.9920909 & & & \\
\hline 19080 & 3.46E-04 & 8.89E-03 & $6.14 \mathrm{E}-04$ & -3.246905231 & $\mathrm{k}$ & 3.93E-03 & $-2.38 \mathrm{E}-04$ \\
\hline 21360 & $3.74 \mathrm{E}-04$ & $8.91 \mathrm{E}-03$ & 5.86E-04 & -3.170098481 & & & \\
\hline 23640 & 3.36E-04 & $8.88 \mathrm{E}-03$ & $6.24 \mathrm{E}-04$ & -3.273995124 & & & \\
\hline 25140 & 3.84E-04 & 8.92E-03 & 5.76E-04 & -3.145857003 & & & \\
\hline 26640 & 3.65E-04 & 8.90E-03 & 5.95E-04 & -3.194996478 & & & \\
\hline 28980 & 3.55E-04 & $8.90 \mathrm{E}-03$ & 6.05E-04 & -3.220586073 & & & \\
\hline 31980 & $2.88 \mathrm{E}-04$ & 8.83E-03 & $6.72 \mathrm{E}-04$ & -3.422723287 & & & \\
\hline 32760 & $2.50 \mathrm{E}-04$ & 8.79E-03 & $7.10 \mathrm{E}-04$ & -3.561464847 & & & \\
\hline 34260 & $2.21 \mathrm{E}-04$ & 8.76E-03 & 7.39E-04 & -3.68078519 & & & \\
\hline 36540 & 2.21E-04 & 8.76E-03 & 7.39E-04 & -3.68078519 & & & \\
\hline 38820 & 1.73E-04 & 8.71E-03 & 7.87E-04 & -3.920413631 & & & \\
\hline
\end{tabular}




\section{$40{ }^{\circ} \mathrm{C}$}

$\begin{array}{ll}{[\mathrm{Me}]_{0}} & 1.40 \mathrm{E}-03 \mathrm{M} \\ \text { [amine }]_{0} & 9.50 \mathrm{E}-03 \mathrm{M}\end{array}$

Time / s [Me ester] / M [amine] / M [pdt] / M $\ln \left(\left([\text { Me ester }]_{0}-\mathrm{x}\right) /\left(\left([a m i n e]_{0}-\mathrm{x}\right)\right)\right.$

$\begin{array}{rrrrr}600 & 1.34 \mathrm{E}-03 & 9.44 \mathrm{E}-03 & 6.01 \mathrm{E}-05 & -1.949953138 \\ 1740 & 1.26 \mathrm{E}-03 & 9.36 \mathrm{E}-03 & 1.40 \mathrm{E}-04 & -2.002980376 \\ 2880 & 1.12 \mathrm{E}-03 & 9.22 \mathrm{E}-03 & 2.81 \mathrm{E}-04 & -2.105657384 \\ 3960 & 1.12 \mathrm{E}-03 & 9.22 \mathrm{E}-03 & 2.81 \mathrm{E}-04 & -2.105657384 \\ 5100 & 9.82 \mathrm{E}-04 & 9.08 \mathrm{E}-03 & 4.21 \mathrm{E}-04 & -2.223851052 \\ 6180 & 9.42 \mathrm{E}-04 & 9.04 \mathrm{E}-03 & 4.61 \mathrm{E}-04 & -2.261097984 \\ 7680 & 8.42 \mathrm{E}-04 & 8.94 \mathrm{E}-03 & 5.61 \mathrm{E}-04 & -2.362425093 \\ 8400 & 8.42 \mathrm{E}-04 & 8.94 \mathrm{E}-03 & 5.61 \mathrm{E}-04 & -2.362425093 \\ 9480 & 8.22 \mathrm{E}-04 & 8.92 \mathrm{E}-03 & 5.81 \mathrm{E}-04 & -2.384277471 \\ 10620 & 7.42 \mathrm{E}-04 & 8.84 \mathrm{E}-03 & 6.62 \mathrm{E}-04 & -2.47790018 \\ 11700 & 7.22 \mathrm{E}-04 & 8.82 \mathrm{E}-03 & 6.82 \mathrm{E}-04 & -2.50302849 \\ 12780 & 6.82 \mathrm{E}-04 & 8.78 \mathrm{E}-03 & 7.22 \mathrm{E}-04 & -2.555630049 \\ 13920 & 5.81 \mathrm{E}-04 & 8.68 \mathrm{E}-03 & 8.22 \mathrm{E}-04 & -2.703210924 \\ 15000 & 5.01 \mathrm{E}-04 & 8.60 \mathrm{E}-03 & 9.02 \mathrm{E}-04 & -2.842347967 \\ 16140 & 5.01 \mathrm{E}-04 & 8.60 \mathrm{E}-03 & 9.02 \mathrm{E}-04 & -2.842347967 \\ 17220 & 4.41 \mathrm{E}-04 & 8.54 \mathrm{E}-03 & 9.62 \mathrm{E}-04 & -2.963162124\end{array}$

\section{$45^{\circ} \mathrm{C}$}

$[\mathrm{Me}]_{0}$

[amine $]_{0}$

Time / s

[Me ester] / $\mathrm{M}$ $1.43 \mathrm{E}-03$

$1.26 \mathrm{E}-03$

$1.10 \mathrm{E}-03$

9.38E-04

$8.98 \mathrm{E}-04$

$6.94 \mathrm{E}-04$

$6.12 \mathrm{E}-04$

$5.10 \mathrm{E}-04$

$4.90 \mathrm{E}-04$

3.67E-04

3.67E-04

3.06E-04

2.86E-04

2.45E-04

2.24E-04

$1.63 \mathrm{E}-04$

$1.43 \mathrm{E}-04$

$1.84 \mathrm{E}-04$

$1.22 \mathrm{E}-04$

$1.02 \mathrm{E}-04$

$6.12 \mathrm{E}-05$

$4.08 \mathrm{E}-05$

$6.12 \mathrm{E}-05$

$6.12 \mathrm{E}-05$

$0.00 \mathrm{E}+00$

$0.00 \mathrm{E}+00$
1.59E-03 M

9.50E-03 M

[amine] / M $\quad[\mathrm{pdt}] / \mathrm{M} \quad \operatorname{In}\left(\left([\mathrm{Me} \text { ester }]_{0}-\mathrm{x}\right) /\left(\left([a m i n e]_{0}-\mathrm{x}\right)\right)\right.$ $-1.877631308$ $-1.981357357$

$-2.101556309$

$-2.24361942$

$-2.283448607$

$-2.517838777$

$-2.633470323$

$-2.803748184$

$-2.842143936$

$-3.115143612$

$-3.115143612$

$-3.290042332$

$-3.35654863$

$-3.505707536$

$-3.59021365$

$-3.901113715$

$-4.032114486$

$-3.785854913$

$-4.183728123$

$-4.363506185$

$-4.869225328$

$-5.272127383$

$-4.869225328$

$-4.869225328$

\#NUM!

7.97E-03 1.53E-03

$7.91 \mathrm{E}-03 \quad 1.59 \mathrm{E}-03$

7.91E-03 1.59E-03

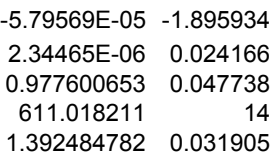

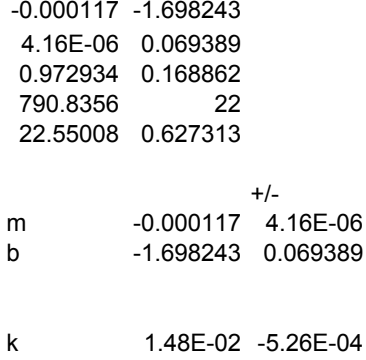




\section{$50{ }^{\circ} \mathrm{C}$}

$\begin{array}{ll}{[\mathrm{Me}]_{0}} & 1.11 \mathrm{E}-03 \mathrm{M} \\ {[\mathrm{amine}]_{0}} & 9.50 \mathrm{E}-03 \mathrm{M}\end{array}$

Time / s [Me ester] / M [amine] / M [pdt] / M $\quad \ln \left(\left([\text { Me ester }]_{0}-\mathrm{x}\right) /\left(\left([a m i n e]_{0}-\mathrm{x}\right)\right)\right.$

$\begin{array}{rllll}540 & 9.57 \mathrm{E}-04 & 9.35 \mathrm{E}-03 & 1.51 \mathrm{E}-04 & -2.278831513 \\ 1320 & 7.68 \mathrm{E}-04 & 9.16 \mathrm{E}-03 & 3.40 \mathrm{E}-04 & -2.478272672 \\ 1920 & 6.17 \mathrm{E}-04 & 9.01 \mathrm{E}-03 & 4.91 \mathrm{E}-04 & -2.680685996 \\ 2520 & 5.04 \mathrm{E}-04 & 8.90 \mathrm{E}-03 & 6.05 \mathrm{E}-04 & -2.870962387 \\ 3060 & 5.04 \mathrm{E}-04 & 8.90 \mathrm{E}-03 & 6.05 \mathrm{E}-04 & -2.870962387 \\ 3660 & 4.16 \mathrm{E}-04 & 8.81 \mathrm{E}-03 & 6.93 \mathrm{E}-04 & -3.053372053 \\ 4260 & 3.78 \mathrm{E}-04 & 8.77 \mathrm{E}-03 & 7.31 \mathrm{E}-04 & -3.144382137 \\ 4800 & 3.28 \mathrm{E}-04 & 8.72 \mathrm{E}-03 & 7.81 \mathrm{E}-04 & -3.281720605 \\ 5400 & 3.15 \mathrm{E}-04 & 8.71 \mathrm{E}-03 & 7.94 \mathrm{E}-04 & -3.319495521 \\ 6000 & 3.02 \mathrm{E}-04 & 8.69 \mathrm{E}-03 & 8.06 \mathrm{E}-04 & -3.358869624 \\ 6600 & 2.27 \mathrm{E}-04 & 8.62 \mathrm{E}-03 & 8.82 \mathrm{E}-04 & -3.637820051 \\ 7140 & 1.64 \mathrm{E}-04 & 8.56 \mathrm{E}-03 & 9.45 \mathrm{E}-04 & -3.955907383 \\ 7740 & 1.76 \mathrm{E}-04 & 8.57 \mathrm{E}-03 & 9.32 \mathrm{E}-04 & -3.883270735 \\ 8340 & 1.64 \mathrm{E}-04 & 8.56 \mathrm{E}-03 & 9.45 \mathrm{E}-04 & -3.955907383 \\ 8880 & 1.51 \mathrm{E}-04 & 8.54 \mathrm{E}-03 & 9.57 \mathrm{E}-04 & -4.034476599 \\ 9480 & 1.20 \mathrm{E}-04 & 8.51 \mathrm{E}-03 & 9.89 \mathrm{E}-04 & -4.264398192 \\ 10080 & 9.70 \mathrm{E}-05 & 8.49 \mathrm{E}-03 & 1.01 \mathrm{E}-03 & -4.471802045 \\ 10620 & 7.68 \mathrm{E}-05 & 8.47 \mathrm{E}-03 & 1.03 \mathrm{E}-03 & -4.702356399\end{array}$

\section{$55^{\circ} \mathrm{C}$}

$\begin{array}{ll}{[\mathrm{Me}]_{0}} & 1.02 \mathrm{E}-03 \mathrm{M} \\ {[\mathrm{amine}]_{0}} & 9.50 \mathrm{E}-03 \mathrm{M}\end{array}$

Time / s [Me ester] / M [amine] / M [pdt] / M $\operatorname{In}\left(\left([\text { Me ester }]_{0}-\mathrm{x}\right) /\left(\left([a m i n e]_{0}-\mathrm{x}\right)\right)\right.$

$\begin{array}{rlll}720 & 7.75 \mathrm{E}-04 & 9.25 \mathrm{E}-03 & 2.50 \mathrm{E}-04 \\ 1440 & 6.04 \mathrm{E}-04 & 9.08 \mathrm{E}-03 & 4.20 \mathrm{E}-04 \\ 2100 & 4.34 \mathrm{E}-04 & 8.91 \mathrm{E}-03 & 5.91 \mathrm{E}-04 \\ 2760 & 3.15 \mathrm{E}-04 & 8.79 \mathrm{E}-03 & 7.09 \mathrm{E}-04 \\ 3360 & 3.55 \mathrm{E}-04 & 8.83 \mathrm{E}-03 & 6.70 \mathrm{E}-04 \\ 4020 & 2.36 \mathrm{E}-04 & 8.71 \mathrm{E}-03 & 7.88 \mathrm{E}-04 \\ 4680 & 1.97 \mathrm{E}-04 & 8.67 \mathrm{E}-03 & 8.28 \mathrm{E}-04 \\ 5280 & 1.31 \mathrm{E}-04 & 8.61 \mathrm{E}-03 & 8.93 \mathrm{E}-04 \\ 5940 & 1.05 \mathrm{E}-04 & 8.58 \mathrm{E}-03 & 9.20 \mathrm{E}-04 \\ 6540 & 3.94 \mathrm{E}-05 & 8.51 \mathrm{E}-03 & 9.85 \mathrm{E}-04 \\ 7200 & 0.00 \mathrm{E}+00 & 8.48 \mathrm{E}-03 & 1.02 \mathrm{E}-03\end{array}$

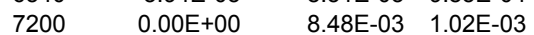

$-2.479326136$

$-2.709585254$

$-3.022728218$

$-3.327819912$

$-3.214510756$

$-3.606493726$

$-3.784280539$

$-4.182141741$

$-4.402227467$

$-5.375370996$

\#NUM!

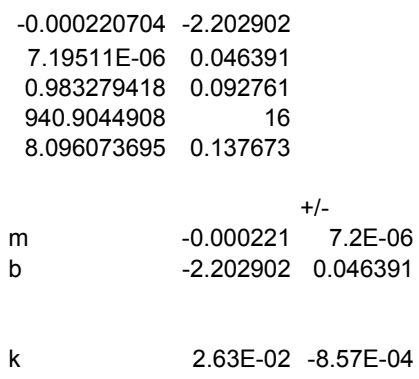


2nd order rate law plots

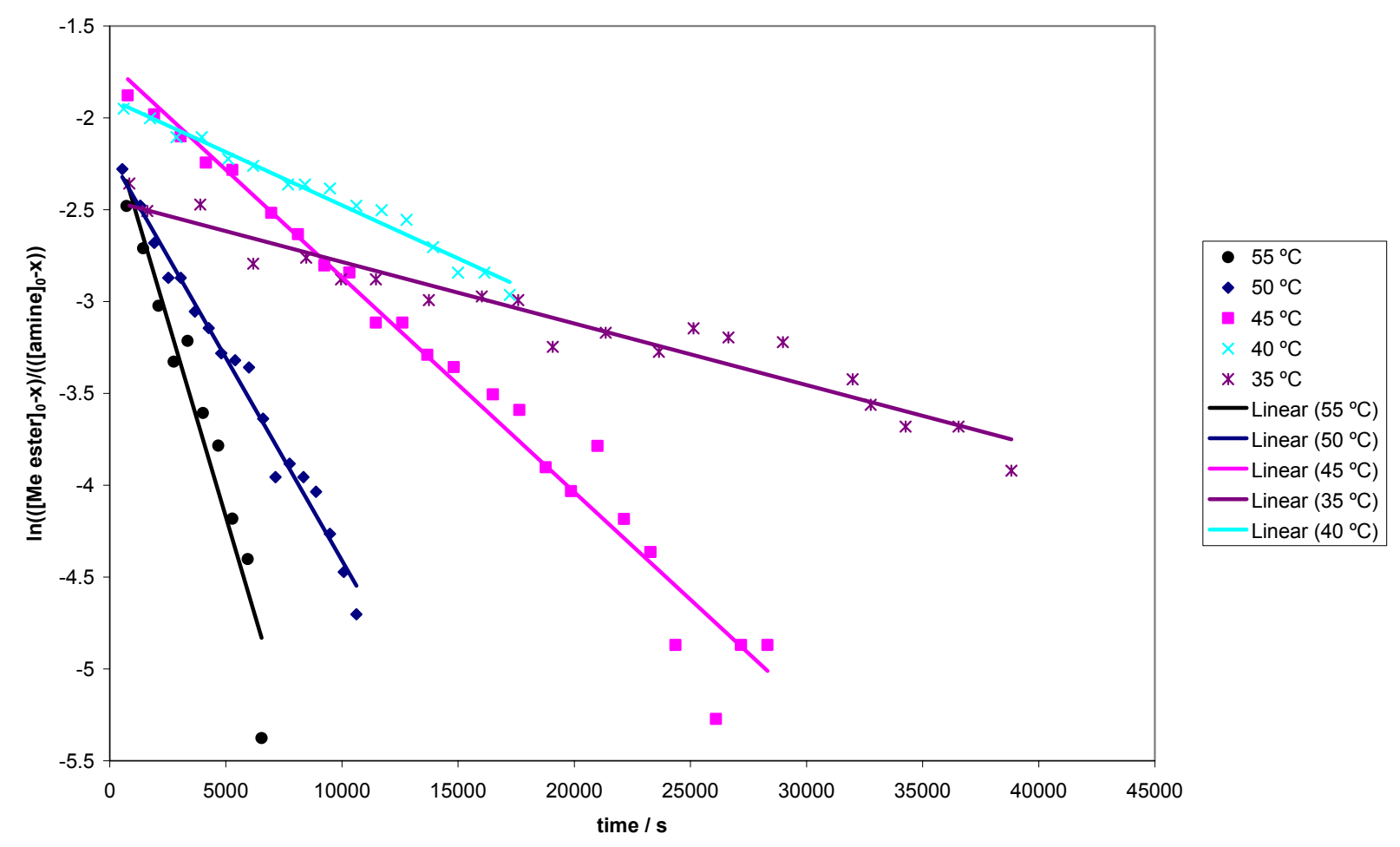

\section{Eyring Plot Data}

\begin{tabular}{cc}
$\mathrm{T} / \mathrm{K}$ & \multicolumn{2}{c}{$\mathrm{k} / \mathrm{M}^{-1} \mathrm{~s}^{-1}$} \\
308 & $3.93 \mathrm{E}-03$ \\
313 & $7.16 \mathrm{E}-03$ \\
318 & $1.48 \mathrm{E}-02$ \\
323 & $2.63 \mathrm{E}-02$ \\
328 & $5.04 \mathrm{E}-02$
\end{tabular}

$$
\begin{aligned}
1 / \mathrm{T} / \mathrm{K}^{-1} & \ln (\mathrm{k} / \mathrm{T}) \\
0.003247 & -11.26922 \\
0.003195 & -10.68545 \\
0.003145 & -9.975179 \\
0.003096 & -9.415839 \\
0.003049 & -8.780778
\end{aligned}
$$

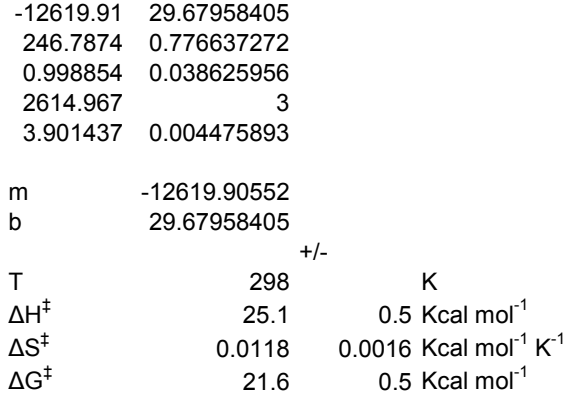


Eyring Plot

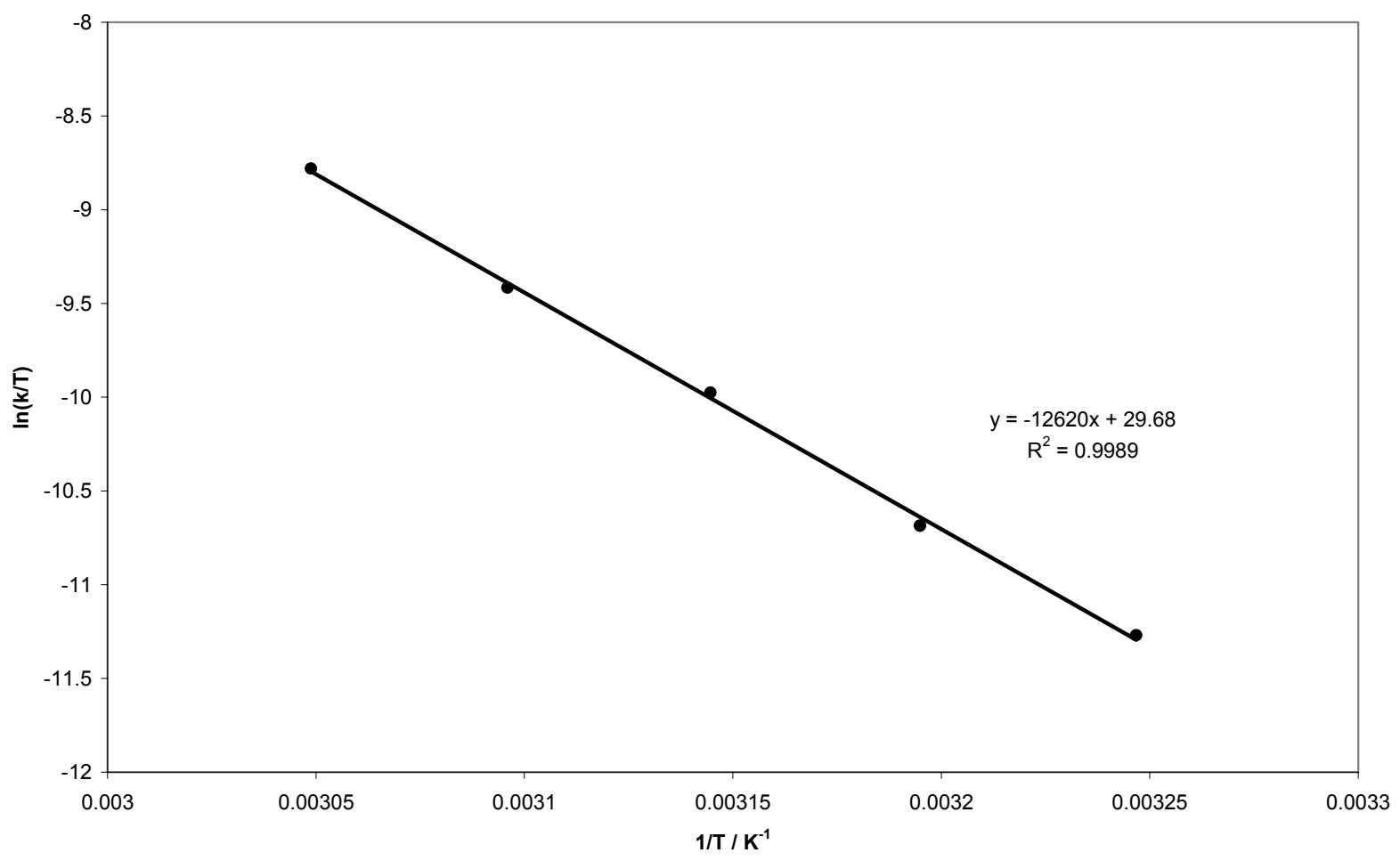

S-13 